\title{
Synthesis and Characterization of Some New 4,5-Dihydropyrazolyl Thiazoles
}

\author{
FAROUQ E. HAWAIZ \\ Department of Chemistry, College of Education, University of Salahaddin-Hawler, \\ Kurdistan Region, Iraq \\ farouqemam@yahoo.com
}

Received 18 July 2014 / Accepted 26 July 2014

\begin{abstract}
The prepared starting material 2,4-bis (4-chlorobenzyloxy)acetophenone (1) has been reacted with different substituted benzaldehydes to give a series of new chalcones (2a-j). The prepared new chalcones were subjected to react with thiosemicarbazide according to the Michael addition reaction to afford new thiocarbamoylpyrazoline derivatives (3a-j). A thiocarbamoyl group in compounds (3a-j) was cyclized with $p$-bromophenacyl bromide to give a series of new 4,5-dihydropyrazolyl thiazoles $(\mathbf{4 a - j})$. The structures of the synthesized compounds were characterized by spectral methods: FT-IR, ${ }^{1} \mathrm{H}-\mathrm{NMR},{ }^{13} \mathrm{C}-\mathrm{NMR}$ and DEPT-135 spectra.
\end{abstract}

Keywords: Chalcones, Benzylation, Pyrazoline, Thiazole, Thiosemicarbazide

\section{Introduction}

Thiosemicarbazide is one of the most important precursor for the formation of different acyclic $^{1}$ and cyclic organic compounds, especially heterocyclic compounds containing nitrogen and sulfur heteroatoms ${ }^{2}$. Chalcones also can be used as a very useful precursor besides thiosemicarbazide and other reagents for the formation of different important heterocyclic compounds such as pyrazolines ${ }^{3}$, thiadiazoles ${ }^{4}$ and thiazepines ${ }^{5}$. Chalcones constitute a class of naturally occurring and synthetic compounds belonging to the flavonoid family ${ }^{6}$. It has been reported that chalcones, pyrazoline and thiazoles possess a wide spectrum of biological activity which include anti-malarial ${ }^{7}$, anti-inflammatory ${ }^{8}$, analgesic ${ }^{9}$, antioxidant ${ }^{10}$ and other antimicrobial activities ${ }^{11}$. The present investigation describes the synthesis and spectroscopic studies of some new compounds containing two different kinds of heterocyclic moieties, pyrazoline and thiazoles.

\section{Experimental}

Melting points were determined using a Gallen Kamp electrothermal melting point apparatus. IR spectra were recorded on FTIR-Thermo-Mattson-300 using $\mathrm{KBr}$ disc. ${ }^{1} \mathrm{H}$ NMR, DEPT-135 and ${ }^{13} \mathrm{C}$ NMR spectra were recorded on a Bruker(300MHz) with TMS as internal reference. 


\section{Preparation of 2,4-bis (4-chlorobenzyloxy) acetophenone (1)}

The starting material (1) was prepared according to the procedure ${ }^{12}$. Washed several times with water and cold ethanol, dried and recrystallized from ethanol to obtain white crystals of 2,4-bis(4-chlorobenzyloxy)acetophenone (1): m.p. (106-107 $\left.{ }^{\circ} \mathrm{C}\right)$, yield $(6 \mathrm{~g}, 61 \%)$.

FT-IR $\left(\mathrm{cm}^{-1}\right): 3093(\mathrm{C}-\mathrm{H}$ aromatic), 2933, 2880(C-H aliphatic), 1662(C=O), 1595, 1567(C=C), 1265(C-O). ${ }^{1} \mathrm{H}$ NMR(ppm): 2.45(s, 3H, $\left.\mathrm{CH}_{3}\right), 5.19\left(\mathrm{~s}, 2 \mathrm{H}, \mathrm{CH}_{2}-\mathrm{Bz}^{\prime}\right), 5.23(\mathrm{~s}, 2 \mathrm{H}$, $\left.\mathrm{CH}_{2}-\mathrm{Bz}\right), 6.68\left(\mathrm{~d}, 1 \mathrm{H}, \mathrm{Ar}-\mathrm{H}_{5}\right), 6.8\left(\mathrm{~s}, 1 \mathrm{H}, \mathrm{H}_{3}\right) ; 7.46-7.54(\mathrm{~m}, 8 \mathrm{H}, 2 \mathrm{Ar}), 7.69\left(\mathrm{~d}, 1 \mathrm{H}, \mathrm{H}_{6}\right)$.

${ }^{13} \mathrm{C} \mathrm{NMR}(\mathrm{ppm}): 32.23\left(\mathrm{CH}_{3}\right), 69.21(\mathrm{Bz}), 69.79\left(\mathrm{Bz}^{\prime}\right), 100.01\left(\mathrm{C}_{3}\right), 107.38\left(\mathrm{C}_{5}\right), 121.45\left(\mathrm{C}_{1}\right)$, $128.9\left(\mathrm{C}_{2}\right), \quad 130.15\left(\mathrm{C}_{3}\right), \quad 132.3\left(\mathrm{C}_{6}\right), \quad 133.8\left(\mathrm{C}_{4}\right), \quad 135.88\left(\mathrm{C}_{1} \cdot\right), \quad 160.00\left(\mathrm{C}_{2}\right), \quad 163.38\left(\mathrm{C}_{4}\right)$, 196.67(C=O).

Synthesis of 1-(2,4-bis(4-chlorobenzyloxy)phenyl)-3-(substitutedphenyl)prop-2-en1 -one $(2 a-j)^{13}$

The prepared 2,4-bis(4-chlorobenzyloxy)acetophenone (1) $(0.02 \mathrm{~mol})$ was dissolved in ethanol $(5 \mathrm{~mL})$ and added to the solution of $(0.02 \mathrm{~mol})$ of substituted benzaldehydes in ethanol $(5 \mathrm{~mL})$ and $2 \mathrm{~mL}$ of $(4 \%$ ethanolic $\mathrm{NaOH})$. The mixture was stirred at room temperature for $(5-10 \mathrm{~min})$. The mixture was solidified and the pale yellow chalcones were separated by suction filtration, washed with ethanol and water to neutralize, dried and purified by recrystallization from a suitable solvent (toluene or ethanol), the results are summarized in Table 1.

Table 1. Some physical properties of the synthesized chalcones (2a-j), pyrazolines (3a-j) and thiazoles $(\mathbf{4 a - j})$

\begin{tabular}{cccccccccccc}
\hline & & \multicolumn{3}{c}{ Chalcones (2a-j) } & \multicolumn{3}{c}{ Pyrazolines (3a-j) } & \multicolumn{3}{c}{ Thiazoles (4a-j) } \\
\cline { 2 - 10 } & Products & M.P & $\%$ & $\begin{array}{c}\text { Time } \\
\text { min. }\end{array}$ & M.P & $\%$ & $\begin{array}{c}\text { Time } \\
\text { h. }\end{array}$ & M.P & $\%$ & $\begin{array}{c}\text { Time } \\
\text { h. }\end{array}$ \\
\hline a & $3-\mathrm{Bz}$ & $128-130$ & 95 & 5 & $168-170$ & 76 & 5 & $184-186$ & 80 & 2.5 \\
$\mathrm{~b}$ & $4-\mathrm{CH}_{3}$ & $123-125$ & 97 & 5 & $164-166$ & 65 & 6 & $179-181$ & 94 & 2 \\
$\mathrm{c}$ & $4-\mathrm{F}$ & $110-112$ & 99 & 5 & $174-176$ & 85 & 5 & $170-172$ & 83 & 2 \\
$\mathrm{~d}$ & $4-\mathrm{Bz}$ & $178-180$ & 94 & 5 & $169-171$ & 60 & 6 & $177-179$ & 88 & 2.5 \\
$\mathrm{e}$ & $2-\mathrm{F}$ & $93-95$ & 77 & 4 & $167-169$ & 65 & 5 & $188-190$ & 78 & 2 \\
$\mathrm{f}$ & $4-\mathrm{N}\left(\mathrm{CH}_{3}\right)_{2}$ & $133-134$ & 82 & 6 & $162-164$ & 78 & 6 & $193-195$ & 73 & 2 \\
$\mathrm{~g}$ & $4-\mathrm{Br}$ & $160-162$ & 97 & 1 & $155-157$ & 96 & 6 & $158-160$ & 50 & 2 \\
$\mathrm{~h}$ & $4-\mathrm{OCH} H_{3}$ & $112-114$ & 80 & 10 & $172-174$ & 83 & 6 & $202-204$ & 87 & 1.45 \\
$\mathrm{i}$ & $2-\mathrm{Cl}$ & $130-132$ & 99 & 1 & $207-209$ & 70 & 6 & $241-243$ & 79 & 2 \\
$\mathrm{j}$ & $\mathrm{H}$ & $107-110$ & 95 & 5 & $245-247$ & 89 & 6 & $199-201$ & 74 & 2 \\
\hline
\end{tabular}

2a: $\operatorname{FT}-\mathrm{IR}\left(\mathrm{cm}^{-1}\right): 1647(\mathrm{C}=\mathrm{O}), 1616,1602(\mathrm{C}=\mathrm{C}) .{ }^{1} \mathrm{H}-\mathrm{NMR}(\mathrm{ppm}): 5.1\left(\mathrm{~s}, 2 \mathrm{H}, \mathrm{CH}_{2}-\mathrm{Bz}\right), 5.06(\mathrm{~s}$, $\left.2 \mathrm{H}, \mathrm{CH}_{2}-\mathrm{Bz}\right), 4.9\left(\mathrm{~s}, 2 \mathrm{H}, \mathrm{CH}_{2}-\mathrm{RBz}\right), 6.61-7.6(\mathrm{~m}, 21 \mathrm{H}, 5 \mathrm{Ar}-$ rings and $\alpha, \beta$ hydrogens $) .{ }^{13} \mathrm{C}-$ NMR(ppm): 69.2, 69.4, 69.5(3Bz), 100.61( $\left.\mathrm{C}_{3}\right), 106.6\left(\mathrm{C}_{5}\right), 114.53\left(\mathrm{C}_{11}\right), 114.58\left(\mathrm{C}_{1}\right), 116.4\left(\mathrm{C}_{13}\right)$, $121.11\left(\mathrm{C}_{8,15}\right), \quad 127.8\left(\mathrm{C}_{14}\right), \quad 128.81\left(\mathrm{C}_{2}\right), \quad 129.28\left(\mathrm{C}_{3}\right), 133.8\left(\mathrm{C}_{6}\right), \quad 134.64\left(\mathrm{C}_{4}\right), \quad 135.26\left(\mathrm{C}_{1^{\prime}}\right)$, 136.74( $\left(\mathrm{C}_{10}\right), 141.68\left(\mathrm{C}_{9}\right), 158.3\left(\mathrm{C}_{12}\right), 159.2\left(\mathrm{C}_{2}\right), 163.38\left(\mathrm{C}_{4}\right), 189.85(\mathrm{C}=\mathrm{O}) .{ }^{13} \mathrm{C}-\mathrm{DEPT}-135(\mathrm{ppm})$ :

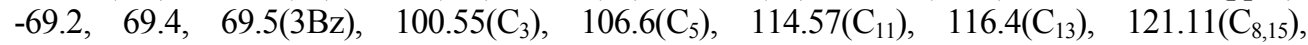
$127.79\left(\mathrm{C}_{14}\right), 128.82\left(\mathrm{C}_{2}\right), 129.28\left(\mathrm{C}_{3}\right), 133.8\left(\mathrm{C}_{6}\right), 141.68\left(\mathrm{C}_{9}\right)$.

2b: FT-IR $\left(\mathrm{cm}^{-1}\right)$ : $1647(\mathrm{C}=\mathrm{O}), \quad 1600,1583(\mathrm{C}=\mathrm{C}) .{ }^{1} \mathrm{H}-\mathrm{NMR}(\mathrm{ppm}): \quad 2.37\left(\mathrm{~s}, \quad 3 \mathrm{H}, \mathrm{CH}_{3}\right)$, 5.07(s, 2H, $\left.\mathrm{CH}_{2}-\mathrm{Bz}\right) 5.08\left(\mathrm{~s}, 2 \mathrm{H}, \mathrm{CH}_{2}-\mathrm{Bz}\right), 6.61-7.85(\mathrm{~m}, 17 \mathrm{H}, 4 \mathrm{Ar}-$ rings and $\alpha, \beta$ hydrogens).

${ }^{13} \mathrm{C}-\mathrm{NMR}(\mathrm{ppm}): 21.5\left(\mathrm{CH}_{3}\right), 69.5(2 \mathrm{Bz}), 100.55\left(\mathrm{C}_{3}\right), 106.55\left(\mathrm{C}_{5}\right), 122.72\left(\mathrm{C}_{1}\right), 126.41\left(\mathrm{C}_{11,15,8}\right)$, 
128.2( $\left(\mathrm{C}_{12,14}\right), 128.8\left(\mathrm{C}_{2}\right), 129.11\left(\mathrm{C}_{3}\right), 133.3\left(\mathrm{C}_{6}\right), 134.11\left(\mathrm{C}_{4}\right), 136.7\left(\mathrm{C}_{10}\right), 140.13\left(\mathrm{C}_{13}\right)$, $140.4\left(\mathrm{C}_{1}\right), \quad 142.22\left(\mathrm{C}_{9}\right), \quad 159.35\left(\mathrm{C}_{2}\right), \quad 162.99\left(\mathrm{C}_{4}\right), \quad 189.85(\mathrm{C}=\mathrm{O}) .{ }^{13} \mathrm{C}-\mathrm{DEPT}-135(\mathrm{ppm})$ : 21.5 $\left(\mathrm{CH}_{3}\right),-69.5(2 \mathrm{Bz}), 100.55\left(\mathrm{C}_{3}\right), 106.55\left(\mathrm{C}_{5}\right), 126.41\left(\mathrm{C}_{11,15,8}\right), 128.2\left(\mathrm{C}_{12,14}\right), 128.8\left(\mathrm{C}_{2}\right)$, $129.11\left(\mathrm{C}_{3}\right), 133.3\left(\mathrm{C}_{6}\right), 142.22\left(\mathrm{C}_{9}\right)$.

Synthesis of 2- Pyrazolines3-(2,4-bis(4-chlorobenzyloxy)phenyl)-5-(substitutedphenyl) -4,5-dihydropyrazole-1-carbothioamide $(\mathbf{3 a}-\mathbf{j})^{14}$

Thiosemicarbazide $(2.27 \mathrm{~g}, 0.025 \mathrm{~mol})$ was added to methanolic suspension $(20 \mathrm{~mL})$ of the prepared chalcones $(0.02 \mathrm{~mol})$ and sodium hydroxide $(0.8 \mathrm{~g}, 0.02 \mathrm{~mol})$. The mixture was heated under reflux with stirring for an appropriate time to complete the reaction and the color was changed from yellow to white indicating the disappearance of the pale yellow chalcones. The pyrazolines (3a-j) were removed by suction filteration, washed with water to neutralize and then with ethanol. The products were dried and recrystallized from ethanol and the results are summarized in Table 1.

3a: $\quad$ FT-IR $\left(\mathrm{cm}^{-1}\right): \quad 3495,3369\left(\mathrm{NH}_{2}\right), \quad 1598,1579(\mathrm{C}=\mathrm{C}, \mathrm{C}=\mathrm{N}), \quad 1350(\mathrm{C}=\mathrm{S}) .{ }^{1} \mathrm{H}-\mathrm{NMR}(\mathrm{ppm})$ : 3.75(dd, $\left.1 \mathrm{H}, \mathrm{H}_{8 \mathrm{a}}\right), 4.7\left(\mathrm{dd}, 1 \mathrm{H}, \mathrm{H}_{8 \mathrm{~b}}\right), 5.1\left(\mathrm{~s}, 2 \mathrm{H}, \mathrm{CH}_{2}-\mathrm{Bz}\right), 5.06\left(\mathrm{~s}, 2 \mathrm{H}, \mathrm{CH}_{2}-\mathrm{Bz}\right), 4.9\left(\mathrm{~s}, 2 \mathrm{H}, \mathrm{CH}_{2}-\right.$ $\mathrm{RBz}), 5.9\left(\mathrm{dd}, 1 \mathrm{H}, \mathrm{H}_{9}\right), 6.5-7.8\left(\mathrm{~m}, 21 \mathrm{H}, 5\right.$ Ar-rings and $\left.\mathrm{NH}_{2}\right) \cdot{ }^{13} \mathrm{C}-\mathrm{NMR}(\mathrm{ppm}): 44.5\left(\mathrm{C}_{8}\right)$, 61.8( $\left(\mathrm{C}_{9}\right), 69.2,69.4,69.5(3 \mathrm{Bz}), 99.56\left(\mathrm{C}_{3}\right), 105.66\left(\mathrm{C}_{5}\right), 110.93\left(\mathrm{C}_{1,11}\right), 112.3\left(\mathrm{C}_{13}\right), 117.03\left(\mathrm{C}_{15}\right)$, 127.5 $\left(\mathrm{C}_{2}\right), \quad 127.71\left(\mathrm{C}_{3}\right), \quad 128.74\left(\mathrm{C}_{14}\right), \quad 129.54\left(\mathrm{C}_{6}\right), \quad 133.42\left(\mathrm{C}_{4}\right), \quad 134.24\left(\mathrm{C}_{1}\right), \quad 154.4\left(\mathrm{C}_{10}\right)$, 155.05( $\left(\mathrm{C}_{7}\right), 157.5\left(\mathrm{C}_{12}\right), 157.68\left(\mathrm{C}_{2}\right), 160.68\left(\mathrm{C}_{4}\right), 183.93(\mathrm{C}=\mathrm{S}) .{ }^{13} \mathrm{C}-\mathrm{DEPT}-135(\mathrm{ppm}):-44.5\left(\mathrm{C}_{8}\right)$, 61.8( $\left(\mathrm{C}_{9}\right),-69.2,69.4,69.5(3 \mathrm{Bz}), 99.56\left(\mathrm{C}_{3}\right), 105.66\left(\mathrm{C}_{5}\right), 110.93\left(\mathrm{C}_{1,11}\right), 112.3\left(\mathrm{C}_{13}\right), 117.03\left(\mathrm{C}_{15}\right)$, 127.5 $\left(\mathrm{C}_{2}\right), 127.71\left(\mathrm{C}_{3}\right), 128.74\left(\mathrm{C}_{14}\right), 129.54\left(\mathrm{C}_{6}\right)$.

3b: $\quad$ FT-IR $\left(\mathrm{cm}^{-1}\right)$ : 3491,3363( $\left.\mathrm{NH}_{2}\right), \quad 1602,1570(\mathrm{C}=\mathrm{C}, \mathrm{C}=\mathrm{N}), \quad$ 1355(C=S). ${ }^{1} \mathrm{H}-\mathrm{NMR}(\mathrm{ppm})$ : 2.3(s, 3H, $\left.\left.\mathrm{CH}_{3}\right), 3.3\left(\mathrm{dd}, 1 \mathrm{H}, \mathrm{H}_{8 \mathrm{a}}\right), 3.9\left(\mathrm{dd}, 1 \mathrm{H}, \mathrm{H}_{8 \mathrm{~b}}\right), 5.12\left(\mathrm{~s}, 2 \mathrm{H}, \mathrm{CH}_{2}-\mathrm{Bz}, \mathrm{Bz}\right)^{\prime}\right), 5.8\left(\mathrm{dd}, 1 \mathrm{H}, \mathrm{H}_{9}\right)$, 6.5-7.8(m, 17H, 4Ar-rings and $\left.\mathrm{NH}_{2}\right){ }^{13} \mathrm{C} \mathrm{NMR}(\mathrm{ppm}): 21.11\left(\mathrm{CH}_{3}\right), 46.07\left(\mathrm{C}_{8}\right), 62.95\left(\mathrm{C}_{9}\right)$, 69.5(2Bz), 100.85( $\left.\mathrm{C}_{3}\right), 106.90\left(\mathrm{C}_{5}\right), 113.71\left(\mathrm{C}_{1}\right), 125.39\left(\mathrm{C}_{11,15}\right), 128.74\left(\mathrm{C}_{2}\right), 128.9\left(\mathrm{C}_{12,14}\right)$, $129.43\left(\mathrm{C}_{3}\right), 130.75\left(\mathrm{C}_{6}\right), 134.55\left(\mathrm{C}_{4}\right), 136.9\left(\mathrm{C}_{13}\right), 137.10\left(\mathrm{C}_{10}\right), 138.9\left(\mathrm{C}_{1}\right), 155.6\left(\mathrm{C}_{7}\right), 158.78\left(\mathrm{C}_{2}\right)$, 161.86( $\left(\mathrm{C}_{4}\right), 173.15(\mathrm{C}=\mathrm{S}) .{ }^{13} \mathrm{C}-\mathrm{DEPT}-135(\mathrm{ppm}): 21.11\left(\mathrm{CH}_{3}\right),-46.07\left(\mathrm{C}_{8}\right), 62.95\left(\mathrm{C}_{9}\right),-69.5(2 \mathrm{Bz})$, $100.85\left(\mathrm{C}_{3}\right), 106.90\left(\mathrm{C}_{5}\right), 125.39\left(\mathrm{C}_{11,15}\right), 128.74\left(\mathrm{C}_{2}\right), 128.9\left(\mathrm{C}_{12,14}\right), 129.43\left(\mathrm{C}_{3^{\prime}}\right), 130.75\left(\mathrm{C}_{6}\right)$.

Synthesis of 4,5-dihydropyrazolyl thiazoles: Synthesis of: 2-(3-(2,4-bis(4chlorobenzyloxy)phenyl)-5-(Substitutedphenyl)-4,5-dihydropyrazol-1-yl)-4-(4bromophenyl)thiazoles $(\mathbf{4 a - j})^{15}$

To a solution of the respective 2-pyrazoline-1-carbothioamides (3a-j) $(0.002 \mathrm{~mol})$ in absolute ethanol $(6 \mathrm{~mL})$, 4-bromophenacyl bromide $(0.56 \mathrm{~g}, 0.002 \mathrm{~mol})$ was added and the mixture was heated under reflux for an appropriate time to complete the reaction. On cooling, the separated solid was filtered, dried and crystallized from the toluene-ethanol, the results are summarized in Table 1.

4a: FT-IR $\left(\mathrm{cm}^{-1}\right): 1604,1585(\mathrm{C}=\mathrm{C}, \mathrm{C}=\mathrm{N}) .{ }^{1} \mathrm{H}-\mathrm{NMR}(\mathrm{ppm}): 3.47\left(\mathrm{dd}, 1 \mathrm{H}, \mathrm{H}_{8 \mathrm{a}}\right), 3.88(\mathrm{dd}, 1 \mathrm{H}$, $\mathrm{H}_{8 \mathrm{~b}}$ ), 5.1(s, 2H, $\left.\mathrm{CH}_{2}-\mathrm{Bz}\right), 5.06\left(\mathrm{~s}, 2 \mathrm{H}, \mathrm{CH}_{2}-\mathrm{Bz}^{\prime}\right), 4.9\left(\mathrm{~s}, 2 \mathrm{H}, \mathrm{CH}_{2}-\mathrm{RBz}\right), 5.52\left(\mathrm{dd}, 1 \mathrm{H}, \mathrm{H}_{9}\right), 6.5-$ $7.8(\mathrm{~m}, \quad 24 \mathrm{H}, \quad 6 \mathrm{Ar}-\mathrm{rings}) .{ }^{13} \mathrm{C}-\mathrm{NMR}(\mathrm{ppm}): 46.7\left(\mathrm{C}_{8}\right), \quad 64.15\left(\mathrm{C}_{9}\right), \quad 69.2, \quad 69.4, \quad 69.5(3 \mathrm{Bz})$, $100.86\left(\mathrm{C}_{3}\right), 103.47\left(\mathrm{C}_{17}\right), 106.83\left(\mathrm{C}_{5}\right), 113.11\left(\mathrm{C}_{1,11}\right), 113.88\left(\mathrm{C}_{13}\right), 119.42\left(\mathrm{C}_{15}\right), 121.42\left(\mathrm{C}_{22}\right)$,

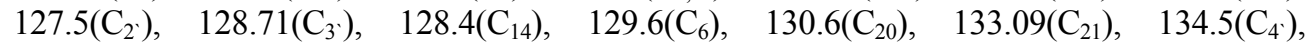
135.79 $\left(\mathrm{C}_{19}\right), 135.39\left(\mathrm{C}_{1}\right), \quad 150.37\left(\mathrm{C}_{10}\right), \quad 151.36\left(\mathrm{C}_{18}\right), \quad 152.9\left(\mathrm{C}_{7}\right), \quad 158.02\left(\mathrm{C}_{12}\right), \quad 158.75\left(\mathrm{C}_{2}\right)$, 161.04( $\left(\mathrm{C}_{4}\right), 164.02\left(\mathrm{C}_{16}\right) .{ }^{13} \mathrm{C}-\mathrm{DEPT}-135(\mathrm{ppm}):-46.7\left(\mathrm{C}_{8}\right), 64.15\left(\mathrm{C}_{9}\right),-69.2,69.4,69.5(3 \mathrm{Bz})$, $100.86\left(\mathrm{C}_{3}\right), 103.47\left(\mathrm{C}_{17}\right), 106.83\left(\mathrm{C}_{5}\right), 113.11\left(\mathrm{C}_{11}\right), 113.88\left(\mathrm{C}_{13}\right), 119.42\left(\mathrm{C}_{15}\right), 121.42\left(\mathrm{C}_{22}\right)$, 127.5 $\left(\mathrm{C}_{2}\right), 128.71\left(\mathrm{C}_{3}\right), 128.4\left(\mathrm{C}_{14}\right), 129.6\left(\mathrm{C}_{6}\right), 130.6\left(\mathrm{C}_{20}\right), 133.09\left(\mathrm{C}_{21}\right)$. 
4b: FT-IR $\left(\mathrm{cm}^{-1}\right)$ : 1601, $1580(\mathrm{C}=\mathrm{C}, \mathrm{C}=\mathrm{N}) .{ }^{1} \mathrm{H}-\mathrm{NMR}(\mathrm{ppm}): 2.7\left(\mathrm{~s}, 3 \mathrm{H}, \mathrm{CH}_{3}\right), 3.15(\mathrm{dd}, 1 \mathrm{H}$, $\left.\mathrm{H}_{8 \mathrm{a}}\right)$, 3.87(dd, $\left.1 \mathrm{H}, \mathrm{H}_{8 \mathrm{~b}}\right), 5.1\left(\mathrm{~s}, 2 \mathrm{H}, \mathrm{CH}_{2}-\mathrm{Bz}, \mathrm{Bz}^{\prime}\right) ; 5.4\left(\mathrm{dd}, 1 \mathrm{H}, \mathrm{H}_{9}\right), 6.7-7.8(\mathrm{~m}, 20 \mathrm{H}, 5 \mathrm{Ar}-$ rings). ${ }^{13} \mathrm{C}-\mathrm{NMR}(\mathrm{ppm}): 19.5\left(\mathrm{CH}_{3}\right), 43.37\left(\mathrm{C}_{8}\right), 63.94\left(\mathrm{C}_{9}\right), 69.32(2 \mathrm{Bz}), 113.11\left(\mathrm{C}_{3}\right), 113.5\left(\mathrm{C}_{17}\right)$, $113.9\left(\mathrm{C}_{5}\right), 115.9\left(\mathrm{C}_{1}\right), 123.10\left(\mathrm{C}_{22}\right), 126.05\left(\mathrm{C}_{11}, 15\right), 127.5\left(\mathrm{C}_{2}\right), 128.31\left(\mathrm{C}_{12,14}\right), 128.81\left(\mathrm{C}_{20}\right)$, $128.95\left(\mathrm{C}_{3}\right), \quad 129.02\left(\mathrm{C}_{6}\right), \quad 129.9\left(\mathrm{C}_{21}\right), \quad 133.5\left(\mathrm{C}_{4}\right), \quad 135.2\left(\mathrm{C}_{19}\right), 137.2\left(\mathrm{C}_{1}\right), \quad 140.02\left(\mathrm{C}_{13}\right)$, 142.5 $\left(\mathrm{C}_{10}\right), 143.22\left(\mathrm{C}_{18}\right), 152.5\left(\mathrm{C}_{7}\right), 160.2\left(\mathrm{C}_{2}\right), 161.2\left(\mathrm{C}_{4}\right), 163.25\left(\mathrm{C}_{16}\right){ }^{13} \mathrm{C}-\mathrm{DEPT}-135(\mathrm{ppm})$ :

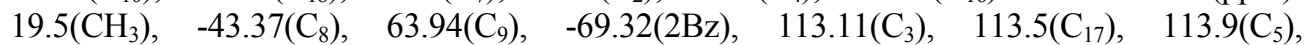
126.05 $\left(\mathrm{C}_{11,15}\right), 127.5\left(\mathrm{C}_{2}\right), 128.31\left(\mathrm{C}_{12,14}\right), 128.81\left(\mathrm{C}_{20}\right), 128.95\left(\mathrm{C}_{3}\right), 129.02\left(\mathrm{C}_{6}\right), 129.9\left(\mathrm{C}_{21}\right)$.

\section{Results and Discussion}

The synthetic process of the newly target molecules 4, 5-dihydropyrazolyl thiazoles is outlined in Scheme 1. The formation of the synthesized chalcones (2a-j), pyrazolines (3a-j) and thiazoles (4a-j) were confirmed on the basis of their spectral methods. In the IR spectra of the synthesized chalcones the shifting of the absorption band of carbonyl group to lower wave numberaround $1647 \mathrm{~cm}^{-1}$ is a strong evidence for the formation of $\alpha, \beta$-unsaturated carbonyl group.

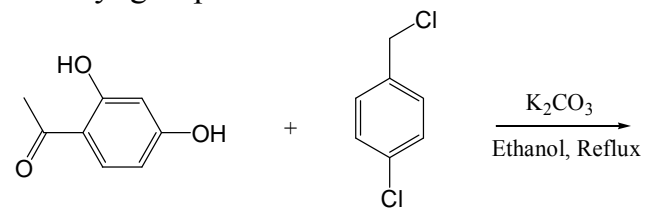<smiles>CC(=O)c1ccc(OCc2ccc(Cl)cc2)cc1OCc1ccc(Cl)cc1</smiles><smiles></smiles><smiles>O=Cc1ccccc1</smiles>
$\mathrm{NaOH}$, Ethanol<smiles>Cc1ccc(Cl)cc1</smiles><smiles>CCC=C(C=CC(=O)c1ccc(OCc2ccc(Cl)cc2)cc1OCc1ccc(Cl)cc1)CCCCCC</smiles><smiles>C1CC2CCC1C2</smiles><smiles>Cc1ccc(Cl)cc1</smiles>
$\mathrm{R}: 3-\mathrm{OCH}_{2} \mathrm{PhCl}$, 4-Me, 4-F, 4-OCH $2 \mathrm{PhCl}, 2-\mathrm{F}, 4-\mathrm{N}\left(\mathrm{CH}_{3}\right)_{2}, 4-\mathrm{Br}, 4-\mathrm{OCH}_{3}$ 2-Cl, H.<smiles>[R]OCc1ccc(Cl)cc1</smiles>
2b: $\mathrm{R}: \mathrm{CH}_{3}$

\section{Scheme 1}


The ${ }^{1} \mathrm{H}$ NMR spectra of chalcones, showed the $\alpha$ - and $\beta$ - protons in aromatic region $\delta$ 6.68.0 which hardly be distinguished from those of the aromatic ring protons. The distinct pick of ${ }^{13} \mathrm{C}$ NMR spectra of $\alpha, \beta$-unsaturated carbonyl group (chalcone) is the $\beta$-carbon atom resonance around $\delta(140)$ downfield to the $\alpha$-carbon atom around $(120 \mathrm{ppm})^{16}$. Further support is come from the DEPT-135 spectra which was used to distinguish between non-protonated carbons, $\mathrm{CH}_{3}, \mathrm{CH}$ and $\mathrm{CH}_{2}$ protons, the DEPT-135 spectra showed a downward signal around $\delta-69$ attributed to benzylic $\mathrm{CH}_{2}$ carbon and the disappearance of non-protonated carbons.

The synthesized pyrazolines (3a-j) were confirmed according their spectral data's. In the IR spectra of pyrazolines, the appearance of a doublet signal at $3495,3369\left(\mathrm{~cm}^{-1}\right)$ attributed to $\mathrm{NH}_{2}$ stretching vibration, and a strong band at 1350 for $\mathrm{C}=\mathrm{S}$ and the disappearance of carbonyl group band at $1647 \mathrm{~cm}^{-1}$ for enone system is a good evidence for the formation of thiocarbamoyl group and the occurrence of cyclization reaction to give 2-pyrazolines. In the ${ }^{1} \mathrm{H}$ NMR spectra of the pyrazolines the protons attached to the $\mathrm{C}_{8}$ and $\mathrm{C}_{9}$ carbon atoms in the 2pyrazoline ring gave an $(\mathrm{ABX})$ spin system ${ }^{17}$ which appeared three doublet to doublets(dd) signals around $\delta 3,4,5.5 \mathrm{ppm}$ for two geminal and one vicinal protons unequivocally prove a 2-pyrazoline structure. Also the appearance of two signals around 40 and $60 \mathrm{ppm}$ in the ${ }^{13} \mathrm{C}$ NMR spectrum, and the appearance of two downward signals at -70 and $-40 \mathrm{ppm}$ attributed to $\mathrm{CH}_{2}$ groups for benzyloxy group and pyrazoline ring respectively. In the DEPT-135 corroborate the 2-pyrazoline structure $^{18}$. The structure of the target molecules 4, 5dihydropyrazolyl thiazoles also confirmed. In the IR spectra the disappearance of $\mathrm{NH}_{2}$ and $\mathrm{C}=\mathrm{S}$ bands considered as a good evidence for the formation of thiazole rings. The ${ }^{1} \mathrm{H}$ NMR Figure 1 showed the $(\mathrm{ABX})$ spin system same as compounds (3a-j). The ${ }^{13} \mathrm{C}$ NMR Figure 2 showed a signal at 103.47 for $\mathrm{C}_{17}$ and a distinct signal for each type of carbons in the molecule. The DEPT-135 Figure 3 perfectly coincided with expected structure.

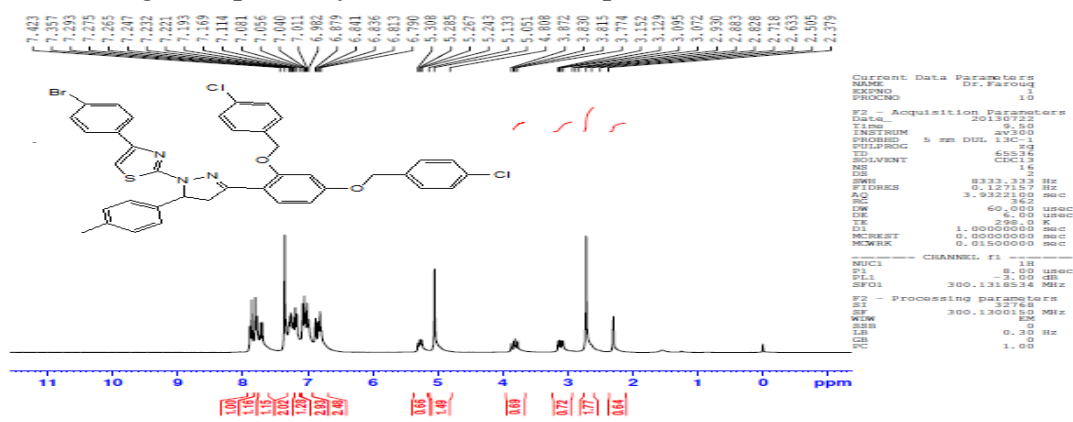

Figure 1. ${ }^{1} \mathrm{H}$ NMR spectrum of compound (4b)

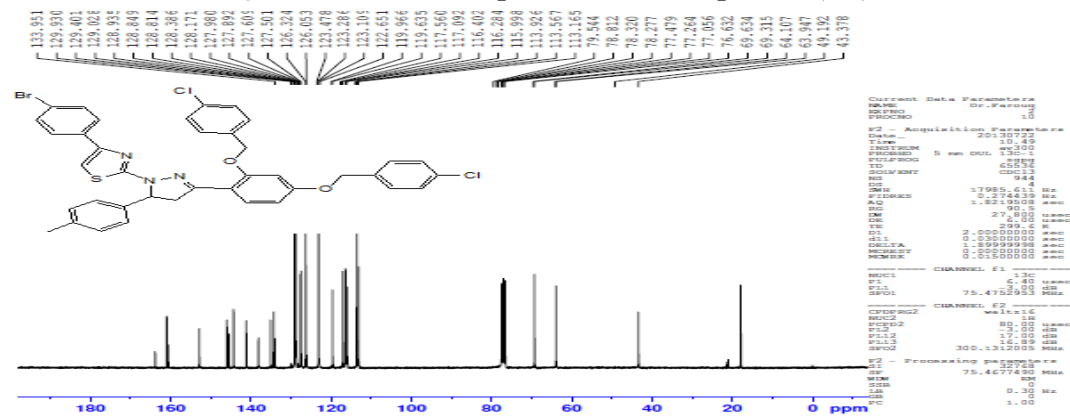

Figure 2. ${ }^{13} \mathrm{C}$ NMR spectrum of compound $(\mathbf{4 b})$ 


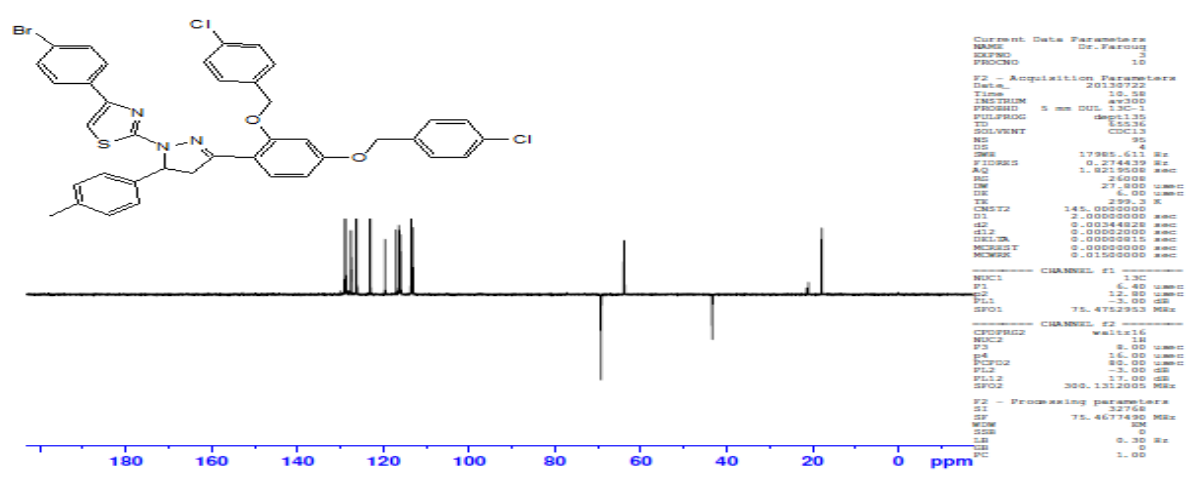

Figure 3. DEPT-135 NMR spectrum of compound (4b)

\section{Conclusion}

The preparation of thiocarbamoylpyrazoline derivatives from substituted chalcones and thiosemicarbazide were achieved in high yields on the basis of Michael addition reaction. A thiocarbamoyl moiety in the synthesized compounds can be used as a precursor group for further preparation such as cyclization with $p$-bromophenacyl bromide to give new 4, 5-dihydropyrazolyl thiazoles.

\section{Acknowledgement}

This study was supported by Chemistry Department, College of Education, Salahaddin University-Hawler, Erbil, Kurdistan region, Iraq.

\section{References}

1. Nevagi R J and Dhake A S, Der Pharma Chemica, 2013, 5(2), 45-49.

2. Raman K and Santosh K S, Int J Chem Sci., 2011, 9(2), 936-940.

3. Sharshira E M and Hamada N M M, Am J Org Chem., 2012, 2(3), 69-73; DOI:10.5923/j.ajoc.20120203.06

4. Vasoya S L, Paghdar D J, Chovatia P T and Joshi H S, J Sci., Islamic Republic Iran, 2005, 16(1), 33-36.

5. Sanjeeva R C, Purnachandra R G, Nagaraj A and Srinivas A, Org Commun., 2008, 1(4), 84-94.

6. Buckingham J E, Dictionary of Natural Products, Champan \& Hall Data Base, CRC Press, 1994.

7. Rongshi Li, Kenyon G L, Fred E Cohen, Xiaowu Chen, Baoqing Gong, Jose N Dominguez, Eugene Davidson, Gary Kurzban, Robert E Miller, Edwin O Nuzum, Philip J Rosenthal and James H McKerrow, J Med Chem., 1995, 38(26), 5031-5037; DOI:10.1021/jm00026a010

8. Sahar M I B, Turk J Chem., 2011, 35, 131-143.

9. Sahu S K, Banerjee M, Samantray A, Behera C and Azam M A, Trop J Pharm Res., 2008, 7(2), 961-968.

10.. Suthakaran R, Somasekhar G, Sredivi C H, Marikannan M, Suganathi K and Nagarajan G, Asian J Chem., 2007, 19(5), 3353-3362.

11. Pravin C M, Kamlesh S V, Rahul P J and Vivek D B, J Korean Chem Soc., 2011, 55(5), 882-886; DOI:10.5012/jkcs.2011.55.5.882

12. Faiq H S H, Farouq E H and Hashim J A, Int J Chem Environ Eng., 2013, 4(6), 373-377. 
13. Kalirajan R, Sivakumar S U, Jubie S, Gowramma B and Suresh B, Int J Chem Tech Res., 2009, 1(1), 27-34.

14. Guo H M, Wang LT, Zhang J, Zhao P S and Jian F F, Molecules, 2008, 13, 2039-2048.

15. Mervatm E, Salwa E M, Nadia, A A and Hala B E, Oriental J Chem., 2010, 26(4), 1265-1270.

16. Suvitha S, Siddig I A, Mohammed A A and Syam M, Molecules, 2012, 17(6), 61796195; DOI:10.3390/molecules 17066179

17. Farouq E H, Awaz J H and Mohammed K S, European J Chem., 2014, 5(2), 233-236

18. Farouq E H and Mohammad K S, J Chem., 2012, 9(3), 1613-1622; DOI:10.1155/2012/525940 